\title{
Relação entre velocidade do vento e energia cinética turbulenta em modelos simplificados da camada limite noturna.
}

\author{
Relationship between wind speed and turbulent kinetic energy in simplified \\ models of nocturnal boundary layer.
}

\author{
Rafael Maroneze, Otávio Costa Acevedo, Felipe Denardin Costa \\ Universidade Federal de Santa Maria \\ rafaelmaroneze@gmail.com; otavio@ufsm.br; fdenardin@gmail.com
}

\begin{abstract}
Resumo
A determinação dos fluxos turbulentos em condições muito estáveis é feita geralmente através de parametrizações. Neste trabalho os fluxos turbulentos são estimados, utilizando um modelo simplificado, através de equações prognósticas para intensidade turbulenta, para o fluxo de calor sensível e a variância da temperatura. Os resultados indicam que o modelo é capaz de reproduzir tanto o acoplamento atmosférico quanto a intermitência da turbulência em condições muito estáveis.

Palavras-chave: Turbulência, fluxos, intermitência, camada limite noturna, acoplamento.
\end{abstract}

\begin{abstract}
The determination of the turbulent fluxes in very stable conditions is done, generally, through parameterizations. In this work the turbulent fluxes are estimated, by using a simplified model, through prognostic equations for the turbulent intensity, the sensible heat flux and the temperature variance. The results indicate that the model is able to reproduce both atmospheric coupling and the intermittent character of the turbulence in very stable conditions.

Keywords: Turbulence, flux, intermittent, nocturnal boundary layer, coupling.
\end{abstract}




\section{Introdução}

A estimativa adequada dos fluxos turbulentos em condições muito estáveis ainda é um grande desafio para os modelos numéricos que descrevem o comportamento médio da camada limite estável (CLE). Embora, na média, muitas características do escoamento sejam reproduzidas, a variação intermitente da intensidade da turbulência não é reproduzida por grande parte dos modelos atmosféricos que utilizam teoria $\mathrm{K}$ na estimativa dos fluxos turbulentos na CLE.

Costa et al. (2011) propuseram um modelo simplificado no qual as componentes do fluxo turbulento de momentum são estimadas diretamente a partir da mistura turbulenta local, dada pelo valor local da energia cinética turbulenta (ECT). Todavia, a estimativa do fluxo de energia na forma de calor sensível é feita através da escala de temperatura que é calculada utilizando teoria $\mathrm{K}$.

O fato de parametrizar o fluxo de energia na forma de calor sensível pode reduzir o número de graus de liberdade do sistema, além de limitar o realismo físico do modelo. Portanto, o objetivo principal desse trabalho é implementar duas equação prognóstica, uma para o fluxo de energia na forma de calor sensível, e a outra para a variância da temperatura de modo a acrescentar mais detalhamento físico à solução.

\section{Modelo}

Considerando uma atmosfera seca, livre dos efeitos de advecção e sem divergência de fluxo radiativo, as esquações que descrevem o comportamento médio das variáveis que controlam o escoamento atmosférico podem ser escritas como Costa et al. (2011):

$$
\begin{gathered}
\frac{\partial u}{\partial t}=f\left(v-v_{g}\right)-\frac{\partial \overline{u^{\prime} w^{\prime}}}{\partial t} \\
\frac{\partial v}{\partial t}=f\left(u_{g}-u\right)-\frac{\partial \overline{v^{\prime} w^{\prime}}}{\partial t} \\
\frac{\partial \theta}{\partial t}=-\frac{\partial \overline{w^{\prime} \theta^{\prime}}}{\partial t} \\
\frac{\partial \theta_{g}}{\partial t}=\frac{1}{C_{g}}\left(I_{\downarrow}-\sigma \theta_{g}^{4}-H_{0}\right)-k_{m}\left(\theta_{g}-\theta_{m}\right) \\
\frac{\partial e}{\partial t}=S u_{*}^{2}-R i S u_{*}^{2}+\left[K_{e} \frac{\partial e}{\partial z}\right]-c_{\varepsilon} \frac{u_{*}^{3}}{l_{m}}
\end{gathered}
$$

Os fluxos turbulentos são calculados como: $\overline{u^{\prime} w^{\prime}}=$ $-u_{*}^{2} \cos (\psi)$ e $\overline{u^{\prime} w^{\prime}}=-u_{*}^{2} \cos (\psi)$, onde $\psi$ é a direção do vento. A velocidade de fricção é calculada diretamente a partir da ECT como: $u_{*}^{2}=e / 5,5$ (para maiores informações sobre o modelo ver Costa et al. (2011)). Aqui, o fluxo de energia na forma de calor sensível é calculado prognosticamente, por:

$$
\begin{array}{r}
\frac{\partial \overline{w^{\prime} \theta^{\prime}}}{\partial t}=-\overline{w^{\prime 2}} \frac{\partial \theta}{\partial z}-\frac{\partial}{\partial z}\left[K_{\theta} \frac{\partial \overline{w^{\prime} \theta^{\prime}}}{\partial z}\right]+C_{v} \frac{g}{\Theta} \overline{\theta^{\prime 2}} \\
-2 C_{\overline{w^{\prime} \theta^{\prime}}} l_{m} \frac{u_{m}}{l^{\prime} \theta^{\prime}}
\end{array}
$$

Já, a equação prognóstica para a variância da temperatura é dada por:

$$
\frac{\partial \overline{\theta^{\prime 2}}}{\partial t}=-2 \overline{w^{\prime} \theta^{\prime}} \frac{\partial \theta}{\partial z}-\frac{\partial}{\partial z}\left[K_{\theta} \frac{\partial \overline{\theta^{\prime 2}}}{\partial z}\right]-C_{\overline{\theta^{\prime 2}}} \frac{u_{*}}{l_{m}} \overline{\theta^{\prime 2}}
$$

Nesse trabalho a velocidadede fricção passa a ser relacionada com a ECT através da seguinte relação: $u_{*}^{2}=$ $e / 4,0$ e a variância da velocidade do vento vertical é dada por $\overline{w^{\prime 2}}=1.44 u_{*}^{2}$ e a equação 5 é reescrita como:

$$
\frac{\partial e}{\partial t}=S_{S} u_{*}^{2}+\frac{g}{\Theta} \overline{w^{\prime} \theta^{\prime}}+\left[K_{e} \frac{\partial e}{\partial z}\right]-c_{\varepsilon} \frac{u_{*}^{3}}{l_{m}}
$$

onde $S_{s}$ é dado por:

$$
S_{S}=\frac{\partial}{\partial z}\left[\sqrt{u^{2}+v^{2}}\right]
$$

Os coeficientes de difusão $K_{e}$ e $K_{\theta}$ são dados por

$$
K_{e}=0,00035 \frac{u^{2}+v^{2}}{\sqrt{\left(\frac{g}{\Theta}\right) \frac{\partial \theta}{\partial z}}}
$$

e

$$
K_{\theta}=\frac{\overline{w^{\prime} \theta^{\prime}}}{\frac{\partial \theta}{\partial z}}
$$

\section{Metodologia}

A metodologia empregada para resolver as equações prognósticas (1), (2),(3),(4),(6),(7)e(8) é idêntica à empregada por Costa et al. (2011). Portanto os limites da camada limite são o topo da camada $h$ e a superfície do solo $(z=0)$. Entre esses dois limites são considerado $n$ níveis principais, com o primeiro fixo em $z=5 \mathrm{~m}$ e os outros igualmente espaçados entre este e o topo da camada limite. Para podermos calcular as equações prognósticas para as componentes do vento e para a temperatura neste níveis principais temos que discretizálas. Como essas equações dependem explicitamente da divergências dos fluxos de energia na forma de calor sensível e de momentum temos que estimar seus valores nos $\mathrm{n}$ níveis principais.Para podermos estimar a divergência dos fluxos nos níveis principais, as equações 
prognósticas de ECT e do fluxo de energia na forma de calor sensível terão que ser calculadas nos níveis intermediários $(z i)$, que estão presentes entre os $\mathrm{n}$ níveis principais $(z)$. Os níveis intermediários são definidos como $z i_{n}=\left(z_{n}+z_{n-1}\right) / 2$. A divisão de camada limite em níveis principais e intermediários pode ser vista na figura 1.

O método numérico empregado para resolver as equações prognósticas é o Runge- Kutta de quarta ordem. No topo da camada, as variáveis são assumidas como constantes, $u(t, h)=u_{g}(t, h)=v_{g}, \theta(t, h)=\Theta$, sendo $\Theta=300 \mathrm{~K}$. A temperatura do substrato $\theta_{m}$ é considerada como $285 \mathrm{~K}$. No primeiro nível, a condição inicial para a componente zonal do vento é $u\left(t, z_{1}\right)=0,1 \mathrm{~m} / \mathrm{s}$, sendo assumido que esta varia linearmente entre este nível até o topo da camada. Para a componente meridional, a condição inicial para todos os níveis é $v(t, z)=v_{g}$ $\theta_{g}=\Theta$. A condição inicial para a temperatura do solo e a temperatura potencial do ar em todos os níveis é $\theta_{g}=\Theta$ e $\theta\left(0, z_{n}\right)=\Theta$. Para ECT o valor inicial dado é igual para todos os níveis intermediários, sendo este o valor mínimo que pode ser assumido pela ECT, $e\left(0, z i_{i}\right)=0,0009 m^{2} / s^{2}$.

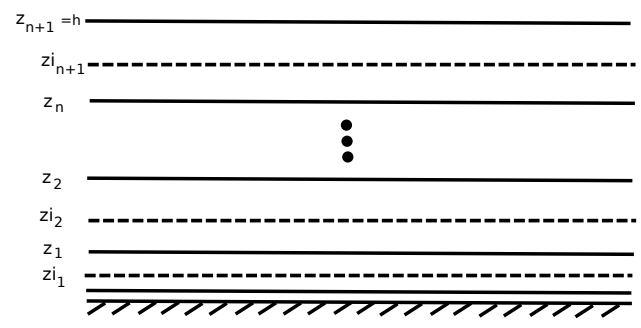

Figura 1: Divisão dos niveis atmosféricos.

\section{Resultados}

Nesta seção, serão apresentados os resultados obtidos nas simulações computacionais com cinco níveis e altura da CLE de $50 \mathrm{~m}$.

A figura 2 apresenta as séries temporais da temperatura do solo (em vermelho) e os níveis atmosférico (demais cores), para diferentes forçantes mecânicos, representados pelo vento geostrófico. Para forçantes mecânicos de baixa e moderada intensidade, nesse caso ventos geostróficos da ordem de $2 \mathrm{~m} / \mathrm{s}$, o modelo reproduz o estado desacoplado, podendo se observar a ocorrência de eventos intermitentes, que causam flutuações instantâneas tanto na temperatura do solo como do ar.

Para forçantes mecânicos intenso (vento geostrófico no topo da CLE),como da ordem de $6 \mathrm{~m} / \mathrm{s}$, o modelo reproduz o estado acoplado. Isso ocorre devido ao forçante mecânico ser suficientemente intenso para manter
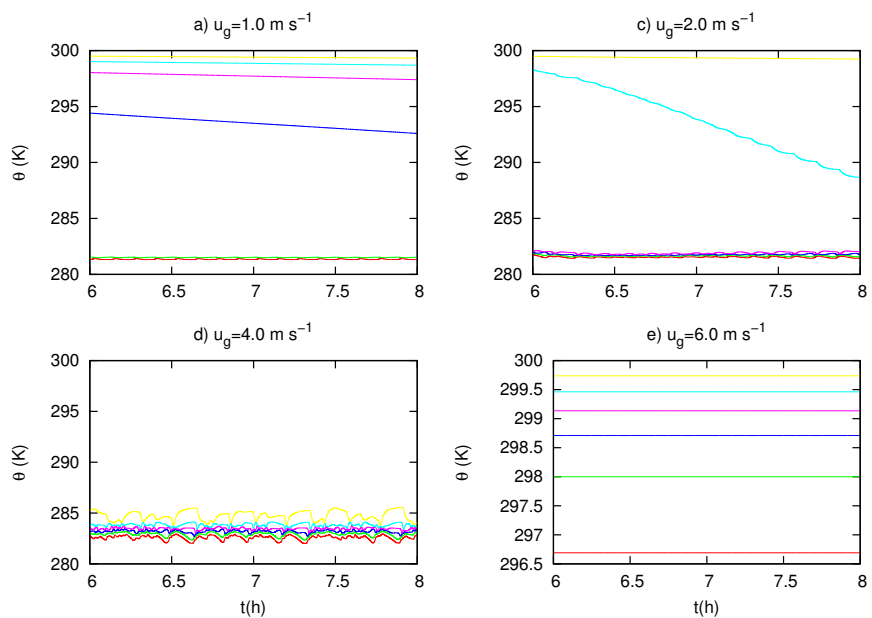

Figura 2: Evolução temporal da temperatura nos diferentes níveis atmosféricos para quatro forçantes mecânicos diferentes. Em cada painel, a linha inferior representa a temperatura do solo, a linha superior é a temperatura no quinto nível atmosférico e as demais linhas representam os demais níveis atmosféricos (linhas inferiores representam níveis inferiores).

a camada turbulenta. Nesse estado, pode se observar que tanto a temperatura do solo como da atmosfera são bem maiores que as simuladas no caso desacoplado, devido à troca turbulenta fazer com que a superfície do solo seja influenciada pelo ar imediatamente acima, mais quente.
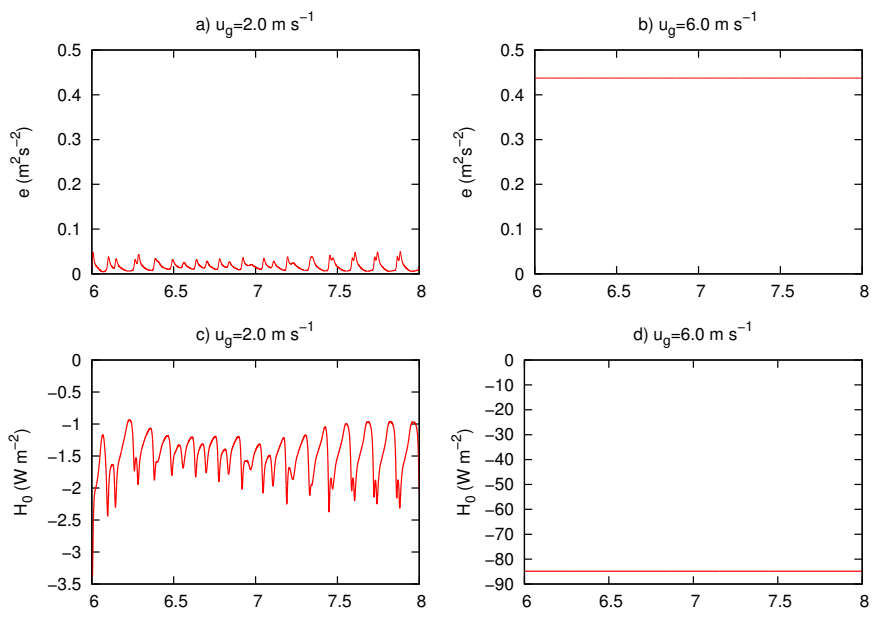

Figura 3: Evoluções temporais da ECT e do fluxo de calor sensível nos primeiro nível atmosférico para dois forçantes mecânicos diferentes

Podemos observar na figura 2 que à medida que 0 vento aumenta, os eventos intermitentes começam a chegar a níveis mais elevados. Logo, os eventos trarão ar quente dos altos níveis para as porções inferiores da atmosfera e jogarão ar frio para cima, simultaneamente. É interessante notar que nos casos analisados na figura 2a) 
os níveis mais altos da atmosfera permanecem não turbulentos. Isto é uma característica do estado desacoplado, pois quando a turbulência se espalha verticalmente por toda a CLE, há o acoplamento e a intermitência não mais ocorre.

O resultado obtido quando velocidade do vento no topo é de $6 \mathrm{~m} / \mathrm{s}$, reproduz uma situação de acoplamento entre a superfície e os níveis mais altos da atmosfera. Isso ocorre porque o forçante mecânico existente é suciente para manter a camada turbulenta em todos os níveis verticais. Nesse estado, pode se observar que tanto a temperatura do solo como a da atmosfera aumentam intensamente, pois as camadas de ar mais quentes em níveis superiores passam a influenciar o que ocorre na superfície. Ao mesmo tempo, os fluxos turbulentos são mais intensos, assim como a própria intensidade da turbulência. Não há oscilações das variáveis nesse caso, como pode ser visto nas figuras 3 e 2

Sun et al. (2012) exploraram a dependência da turbulência com a velocidade média do vento. A escala da velocidade turbulenta é definida como vtke $=\sqrt{e}$ a qual associamos à intensidade da turbulência. Através de dados observacionáis Sun et al. (2012) mostrou a relação entre Vtke e velocidade média do vento horizontal, propondo dois regime, sendo que no primeiro Vtke é pouco intenso e aumenta lentamente com $V$ (regime 1) e o segundo regime Vtke aumenta rapidamente com o $V$ após exceder um valor limite (Regime 2).(Para maiores informações sobre os regimes ver Sun et al. (2012))

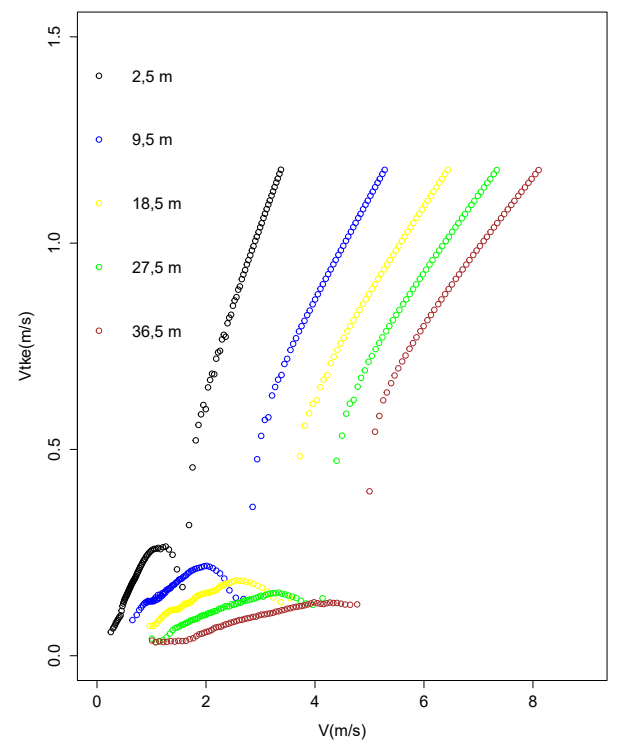

Figura 4: Relação entre Vtke e a velocidade média obtida através do modelo.

A figura 4 apresenta a releção, obtida pelo modelo, ente Vtke e a velocidade horizontal média. Podemos observar que a relação obtida pelo modelo é qualitati- vamente similar a observada por Sun et al. (2012), uma vez que o modelo consegue reproduzir os dois regimes de forma muito semelhante a observada.

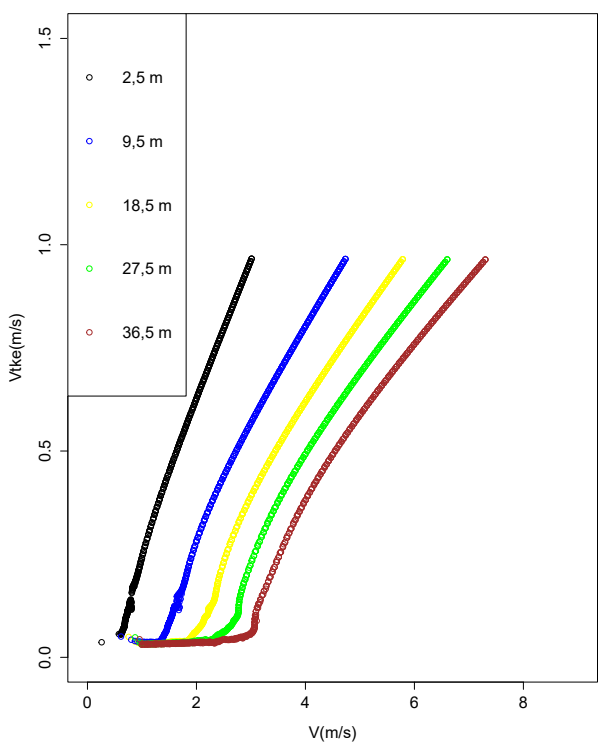

Figura 5: Relação entre Vtke e a velocidade média obtida através do modelo proposto por Costa et al. (2011)

A figura 5 apresenta a releção, obtida pelo modelo proposto por Costa et al. (2011), ente Vtke e a velocidade horizontal média. Podemos observar dois regimes, onde o primeiro regime Vtke é pouco intenso e práticamente constante, já o segundo regime Vtke aumenta rapidamente com o $V$ após excede um valor limite. Portanto, o modelo proposto por Costa et al. (2011) não é capaz de reproduzir o regime 1 descrito por Sun et al. (2012).

\section{Conclusões}

Nesse trabalho foi proposto um modelo para descrever a interação entre a superfície do solo e a atmosfera na camada limite noturna baseado no modelo proposto por Costa et al. (2011). A principal diferença entre os estudos é que neste trabalho o fluxo de energia na forma de calor sensível é estimado através de uma equação prognóstica, enquanto que anteriormente esta quantidade era estimada utilizando a teoria $\mathrm{K}$.

Os resultados obtidos são bastante promissores, pois o modelo consegue reproduzir a transição observada no mundo real entre os estado acoplados e desacoplado. Além disso, o modelo consegue reproduzir eventos intermitentes não periódicos que se assemelham aos observados na camada limite. O modelo consegue reproduzir os resultados similares aos obtidos por Costa et al. (2011), no estado desacoplado, com uma descrição física mais completa. Assim, a inclusão de uma nova equação 
prognóstica manteve os principais resultados daquele modelo, com relações mais realísticas entre as variáveis da camada limite estável.

\section{Referências}

Costa, F. D., Acevedo, O. C., Mombach, J. C. M., Degrazia, G. A. (2011). A simplified model for intermittent turbulence in the nocturnal boundary layer. Journal of the Atmospheric Sciences, 68(8), 1714-1729.

Sun, J., Mahrt, L., ANTA, R. O. M. B., ICHUGINA, Y. E. L. P. (2012). Atmospheric disturbances that generate intermittent turbulence in nocturnal boundary layers. Journal of the Atmospheric Sciences, 69, 279351. 